\title{
Discussion on application status and development trend of electric power dispatching automation system
}

\author{
ZHU Rui ${ }^{1, a}$ \\ ${ }^{1}$ The hulunbuir power supply company, East Inner Mongolia electric power Company Limited, \\ hulunbuir 021008,China \\ aemail: zhurui@126.com
}

\begin{abstract}
Keywords: Electric power dispatching automation system; function; application status; development trend
\end{abstract}

\begin{abstract}
As China's economy has maintained a high growth rate for a long time, the demand for electricity is increasing year by year, so we should pay enough attention to the power dispatch work. Combination in recent years our country electric power dispatch the actual situation and the author for many years to some practical work experience, first briefly describe the functions of power dispatching automation system, then respectively on the present situation of the application of our country and foreign power dispatching automation system are discussed, then the future development trends are discussed in detail, the hope can through ascension of the technological level of China's electric power system of ways to promote the continued development of our national economy.
\end{abstract}

\section{Introduction}

With the continuous development of science and technology in our country, the technology used in the power industry also in the continuous upgrading and due to the mode of supply is from thermal power dominated change for the thermal power, wind power, hydropower and nuclear power the coexistence of diversified supply mode, so that the importance of power transmission become increasingly prominent. Up to now, China has basically achieved the power coverage, and with the gradual improvement of the civilian use of electricity, the power consumption has become the main electricity. In the process of power management, in order to guarantee the electric power safety and the steady supply, we need to conduct real-time monitoring of the power grid, and power dispatching is an important means to realize the real-time data acquisition and monitoring, this method can according to the practice of the regional power grid, electricity, transport power reconfiguration, to the maximum extent so as to ensure the power grid running smoothly.

Rely on the traditional power dispatching management personnel manual management and operation, not only inefficient and prone to misuse, and after integrated automation management systems, power dispatching automation system can remote control, data acquisition, data processing and data analysis and other multiple functions in a body, will be the coverage range electric for real-time monitoring, to provide a strong guarantee for the safe and steady operation of power grid, so that the electric power dispatching technology development direction in the future is the power dispatching automation.

\section{Main functions of power dispatching automation system}

Built on the basis of computer science and technology of automation control system is the electric power dispatching automation system, and with the continuous improvement of the level of science and technology, electric power automation system is gradually perfect and rich.

1) monitoring and control of the power running state

The running state of the power system is mainly through the electric power dispatch automation system is monitored and controlled, so the electricity sector must be strict and effective management of power dispatching automation system, not only to achieve effective real-time 
monitoring of the operation, but also to achieve safety dispatch of power system. Its main function is embodied in two aspects, namely:

Through the intervention of power dispatching automation control system, the system can ensure the safe, efficient and stable operation of power system;

Through the power dispatching automation control system can realize the power system operation status data collection, statistics, analysis and processing and so on, and for all levels of dispatching agencies to present detailed data information. For power system often unexpected, power dispatching automation system can timely analysis and recognition, and take corresponding control measures were preliminary control, at the same time, through the alarm system emergency management personnel to alarm, so that the management staff to quickly adopt follow-up treatment measures.

2) scientific scheduling power

The main task of power dispatching automation system is to meet the safe operation of the power system, to ensure the power quality and to meet the needs of people. Therefore, the power dispatching automation system needs to make a reasonable analysis of the use of electricity, and scientific scheduling, in saving resources, improve the use efficiency, to ensure that residents of electricity.

3) effective control of automatic generation capacity

Due to power dispatching automation system, access to the computer network, and can realize control to the rest of each system, so have the ability to control power to realize closed-loop control ability in the process of power grid operation. In addition, the power dispatching automation system can automatically control the national power grid.

Power dispatching automation system in addition to the above functions, but also a voice alarm function, and the historical trend analysis; sudden event recording function and dispatcher simulation training function.

\section{Application status of power dispatching automation system}

With the rapid development of network technology, computer technology and communication technology, power dispatching automation system has also been greatly improved, the application of a number of new technologies and new equipment, and constantly improve the reliability and applicability of power dispatching automation system. Therefore it has widely used in the power system.

\section{Application of power dispatching automation system in China's electric power system}

At present, most of the power dispatching automation system in our country adopt the internationally accepted standards, such as POSIX operating system and RISC workstation. The commonly used power dispatching automation system is the following:

1) SD-6000 energy management system

The system consists of Zibo Electric Power Bureau of China and Nanjing NARI Group Corporation jointly developed a has a unified supporting platform and open management system, and due to the adoption of the advanced technology of weather satellite imagery, dispatching telephone automatic dialing and ultra large scale scheduling a projection screen, the interface simple, real-time characteristics. At this stage of SD-6000 energy management system in domestic electric power dispatching automation system belongs to one of the leading technology, strong function, high reliability of the products, widely used in the area of Beijing, Nanjing, Henan, Guangdong and Hainan.

2) CC-2000 power dispatching automation system

Developed jointly by Tsinghua University, China Electric Power Research Institute, Beijing Kedong company and group of northeast electric power Quartet of CC-2000 DISPATCHING AUTOMATION of electric power system take the object-oriented technology and distributed structure design, to provide a transparent interface for the application software. In addition CC-2000 DISPATCHING AUTOMATION of electric power system will real-time acquisition of data and a variety of application according to the different functions are stored in different nodes and alpha 
server, so you can appear in some nodes of fault system to ensure the normal operation. The technology through the application in recent years, showing a high versatility, good real-time performance, the technology is mature and reliable has widely used in state power dispatching center, the Northeast Power Grid electroplating Center, North China Power Grid Dispatch Center, Guizhou Power Grid Dispatching Center, to a large extent, guarantee the safety of China's electric power system, a smooth operation.

3) OPEN-2000 energy management system

Nanjing NARI Group in 1998 developed a set of integrated condition monitoring, power control function of dispatcher training, power system application software and distribution management system, has the characteristics of distributed and openness, and is suitable for large and medium-sized, provincial and state of a new generation of energy management system. Due to the technology used in the system is not the latest technology, it has great advantages in the aspects of stability, applicability, maintenance, operation and so on. The system has been in Shanghai, Chongqing, Harbin, Nanjing, Nanning and Yinchuan and other capital cities of the large and medium-sized cities, as well as Jiangsu, Yunnan and Guangxi Province, as well as the adjustment of the province has been applied.

\section{Application of power dispatching automation system in foreign power system}

At the present stage, most of the power dispatching automation system in foreign countries is based on the UNIX operating system, RISC workstation and the relevant international standards:

1) CAE system

The system uses is by Dual Ethernet, customers I server architecture and composed of 64 bit alpha workstation EMS hardware platform using distributed application environment, the integrated DAC, UI, com, Sys, app for a variety of functions in one. Because the function of the system is distributed in each node, the network data can be effectively reduced, which avoids the large amount of the communication bottleneck problem.

2) VALMET system

The system's applicability is very wide, can be connected to ALPHA, IBM, SUN and other workstations, in addition, the system has application software, historical data and real-time data three servers.

3) SPECTRUM system

The system is by Siemens of Germany in the 32 bit sun IBM system workstation hardware platform based on research and development, increase the function of data exchange between the application program and server processes, and because the system adopts the object oriented, distributed component technology. So it is widely used in industrial users, city power company and distribution company.

4) SPIDER system

The system is developed by ABB company, uses the modular structure and distributed database can according to user needs to system configuration. In addition because of the system with the remote signal processing functions, so the only state signal has good stability with a complete maintenance tool.

\section{The future development trend of electric power dispatching automation system}

As in recent years the level of science and technology develops faster and faster, ratio of the application of the advanced technology in the power system also increase rapidly in, a lot of traditional power dispatching automation system is restricted to capacity, transmission speed has also been addressed, so that we continuously improve the technology content of power dispatching automation system. And the gradual improvement of people's material living standards, demand for electricity also in a corresponding increase, so in order to ensure the safety and stability of power grid, we must play a role in the electric power dispatching system, to improve the automation level of power dispatching.

1) the development trend of digital

With the advent of the digital era, the electric power dispatching automation system should be 
developed to the digital direction. The development and research of digital power grid is one of the important research topics in the current electric power industry, China has begun the following several aspects of research on digital, respectively is digital digital management, decision making, communication digital and digital information. The management of digital refers to the realization of digital power system in all aspects of supervision and management, including the data acquisition, analysis, management, network planning, operation and other aspects of decision-making; digital refers to the system to have more data analysis and decision-making ability, which can not only ensure the relevant departments in a timely and effective manner take the corresponding measures, and also to ensure safe and stable operation of the power grid; the digital communication refers to the communication network of digital, this way can not only improve the work efficiency of grid, and can ensure the safety of power grid and fluency; digital information refers to all power related signals converted into digital signals, and digital equipment. The digitization of power dispatching automation system can not only facilitate the management of the power information, but also can make the power information more comprehensive and accurate.

2) multi system operation at the same time

At this stage, China's most commonly used three kinds of power dispatching automation system has different characteristics, so in order to ensure network security, efficient and orderly operation, our center has been achieved multiple systems running at the same time. This way can not only give full play to the characteristics of each different, assure the power system stability, but also can in the single system problems, the rest of the system can still display data from the system, to maintain continued operation in the power system, not as to produce large area power outages, can greatly reduce the economic losses due to accidents.

3) intelligent direction development

Through the integrated scheduling data. The of intelligent analysis, can provide more guarantee for the smooth operation of the power grid, so that one of the main development direction in the future of power dispatching automation system must include the intelligent. Intelligent direction mainly includes the following several aspects, respectively is: the visualization of electric power dispatching; improve the economic benefits of electric power enterprises, optimize the national energy structure; in the event of a sudden, can start emergency emergency control system, in order to control the emergencies. The development of electric power dispatching automation system intelligent results is to establish a comprehensive defense system, intelligent integration of the integrated power management and operation of power and the power dispatching, accelerate the development of electric power dispatching automation system in China.

\section{Conclusion}

In summary, with the continuous development of power industry, demand for electric power dispatching automation system is also more and more high, therefore, we should improve the degree of attention, and through increase technology development efforts, and constantly promote the development of electric power dispatching automation system in China, in order to ensure our country electric power system security, stable and efficient operation at the same time, accelerate the speed of development of national economy.

\section{References}

[1] Pan Honglei. An analysis of the influence of the electric power dispatching automation system on the operation of the power grid.2014 Ningxia electric power [J]., (3).

[2] Zhao Huiru. A discussion on the current situation of the application of power dispatching automation system in our country [J]. building materials and decoration.2013, (12).

[3] Nie Wenbin. On the future development direction of electric power dispatching automation system [J]. modern electronic technology.2012, (16). 